Far Eastern Entomologist

\begin{tabular}{lll}
\hline Number 423: 9-20 & ISSN 1026-051X (print edition) & January 2021 \\
\hline
\end{tabular}

https://doi.org/10.25221/fee.423.3

http://zoobank.org/References/113C8AE8-57CD-4120-A201-69F6266D1B89

\title{
NEW RECORDS OF DIPTERA FROM THE REPUBLIC OF MORDOVIA, RUSSIA
}

\author{
I. MacGowan ${ }^{1)}$, N. E. Vikhrev ${ }^{2)}$, M. G. Krivosheina ${ }^{3)}$, \\ A. B. Ruchin ${ }^{4,}$ ") M. N. Esin ${ }^{4)}$
}

1) National Museums of Scotland, Collection Centre, 242 West Granton Rd, Edinburgh EH5 1JA, Scotland, United Kingdom.

2) Zoological Museum, Moscow State University, Moscow, 125009, Russia.

3) A.N. Severtsov Institute of Ecology and Evolution of RAS, Moscow, 119071, Russia.

4) Joint Directorate of the Mordovia State Nature Reserve and National Park "Smolny», Republic of Mordovia, Saransk, 430005, Russia. *Corresponding author, E-mail: ruchin.alexander@gmail.com

Summary. A list of 55 species of Diptera from families Tanypezidae (1 species), Megamerinidae (1), Acroceridae (1), Psilidae (5), Lonchaeidae (8), Strongylophthalmyiidae (1), Ephydridae (21) Scathophagidae (17 species) collected in the Republic of Mordovia is given. Of them Protearomyia withersi MacGowan, 2014 and Lonchaea baechlii MacGowan, 2016 are recorded from Russia for the first time. Five species, namely Megamerina dolium Fabricius, 1805, Lonchaea carpathica Kovalev, 1974, Ephydra scholtzi Becker, 1896, Strongylophthalmyia pictipes Frey, 1935 and Chamaepsila bicolor (Meigen, 1826), are new for Central part of European Russia. The families Acroceridae, Megamerinidae, Psilidae, Tanypezidae and Strongylophthalmyiidae as well as 53 species are recorded from the Republic of Mordovia for the first time.

Key words: flies, Acroceridae, Ephydridae, Lonchaeidae, Megamerinidae, Psilidae, Scathophagidae, Strongylophthalmyiidae, Tanypezidae, fauna, new records, Europe.

Й. Макгован, Н. Е. Вихрев, М. Г. Кривошеина, А. Б. Ручин, М. Н. Есин. Новые находки двукрылых насекомых (Diptera) в Республике Мордовия, Россия // Дальневосточный энтомолог. 2021. N 423. С. 9-20.

Резюме. Приведен список 55 видов двукрылых семейств Tanypezidae (1 вид), Megamerinidae (1), Acroceridae (1), Psilidae (5), Lonchaeidae (8), Strongylophthalmyiidae (1), Ephydridae (21) и Scathophagidae (17 видов), собранных в Республике Мордовия. Из этих видов новыми для фауны России являются Protearomyia withersi MacGowan, 2014 и Lonchaea baechlii MacGowan, 2016. Впервые для центра европейской части России указываются 5 видов: Megamerina dolium Fabricius, 1805, Lonchaea carpathica Kovalev, 1974, Ephydra scholtzi Becker, 1896, Strongylophthalmyia pictipes Frey, 1935 и Chamaepsila bicolor (Meigen, 1826). Семейства Acroceridae, Megamerinidae, Psilidae Tanypezidae и Strongylophthalmyiidae, а также 53 вида впервые указываются для Мордовии. 


\section{INTRODUCTION}

Diptera is a large order of insects, with at least 150,000 species known worldwide (Courtney et al., 2009). Imagoes and larvae of Diptera are found in a wide variety of terrestrial and aquatic habitats, can live in the tissues of plants and animals, many larvae are coprophages, some larvae are saproxylous (Han \& Choi, 2001; Della Bella et al., 2005; Grichanov \& Khruleva, 2018; Krivosheina \& Krivosheina, 2019; Gornostaev \& Sorokina, 2020; Tiunova \& Makarchenko, 2020). The fly fauna of several regions of the European part of Russia has not been fully identified and catalogued.

For Republic of Mordovia fairly detailed lists of species were published only for families Asilidae (Astakhov et al., 2019), Simuliidae (Budaeva \& Ruchin, 2014), Bombyliidae (Chursina \& Ruchin, 2018a), Syrphidae (Chursina \& Ruchin, 2018b), Limoniidae, Pediciidae and Tipulidae (Pilipenko et al., 2020; Dvorak et al., 2020). The Mordovian fauna of other families of Diptera is still poorly studied.

\section{MATERIAL AND METHODS}

The material for this article was collected in 2019-2020 mainly on the territory of Mordovia State Nature Reserve. We used beer traps (Ruchin et al., 2020) and nets for collecting.

For the general taxonomy and distribution of species the following literature sources were used: Soos (1974); Mathis \& Zatwarnicki (1995); Buck \& Marshall (2006): Shatalkin (2008); Nartshuk (2013); De Jong et al. (2014); MacGowan (2014); Ozerov \& Krivosheina (2015, 2020); MacGowan \& Bächli (2016); Roháček (2016); Ozerov (2019); Krivosheina \& Ozerov (2020). Individual species distribution maps for the Lonchaeidae listed here are available at http://lonchaeidae. myspecies.info/ (MacGowan, 2020). All specimens were identified by I. MacGowan, N.E. Vikhrev, M.G. Krivosheina, A.L. Ozerov and A.I. Shatalkin.

The following abbreviations are used: MSNR - Mordovia State Nature Reserve, distr. district, spec. - specimen. The following abbreviations are used for collectors: AR - A.B. Ruchin, ME - M.N. Esin, KT - K.P. Tomkovich; NV - N.E. Vikhrev; GS - G.B. Semishin. New distributional records are marked with an asterisk: $(*)-$ new from Russia; $\left({ }^{* *}\right)-$ new from the central area of the European part of Russia.

The specimens listed here are deposited either in the Zoological Museum of Moscow University, Russia (those collected by NV and KT) or in the collection of MSNR (those from AR and ME). The specimens of Lonchaeidae are kept in the National Museum of Scotland, Edinburgh.

\section{LIST OF THE SPECIES}

\section{Family Tanypezidae}

\section{Tanypeza longimana Fallen, 1820}

MATERIAL. Russia: Republic of Mordovia, Temnikov distr., MSNR, cordon Novenkovsky $\left(54.931^{\circ} \mathrm{N}, 43.421^{\circ} \mathrm{E}\right), 4-7$. VII $2020,1 \mathrm{spec} ., \mathrm{KT}$.

DISTRIBUTION. Russia (European part). - Holarctic.

\section{Family Megamerinidae}

**Megamerina dolium Fabricius, 1805

MATERIAL. Russia: Republic of Mordovia, Temnikov distr., MSNR, cordon Novenkovsky $\left(54.931^{\circ} \mathrm{N}, 43.421^{\circ} \mathrm{E}\right), 4-7 . \mathrm{VII} 2020,1$ spec., KT.

DISTRIBUTION. Russia (Northern European part, Caucasus, Siberia, Far East). - Europe. 


\section{Family Acroceridae}

\section{Ogcodes gibbosus Linnaeus, 1758}

MATERIAL. Russia: Republic of Mordovia, Temnikov distr., MSNR, cordon Steklyanny $\left(54.894^{\circ} \mathrm{N}, 43.601^{\circ} \mathrm{E}\right), 9-15$.VII $2020,1{ }^{\hat{\gamma}}, \mathrm{KT}$.

DISTRIBUTION. Palaearctic, including North Africa.

\section{Family Lonchaeidae}

\section{Protearomyia nigra (Meigen, 1826)}

MATERIAL. Russia: Republic of Mordovia, Temnikov distr., MSNR, quarter 299 $\left(54.798^{\circ} \mathrm{N}, 43.145^{\circ} \mathrm{E}\right), 9-16 . \mathrm{VII} 2019,1 \mathrm{O}^{\mathrm{O}}$, AR; Rosstanie $\left(54.825^{\circ} \mathrm{N}, 43.137^{\circ} \mathrm{E}\right), 10-19 . \mathrm{VI}$ 2019, 2-9.VII 2019, 2 ô, AR.

DISTRIBUTION. Europe.

*Protearomyia withersi MacGowan, 2014

MATERIAL. Russia: Republic of Mordovia, Temnikov distr., MSNR, quarter 427 $\left(54.736^{\circ} \mathrm{N}, 43.220^{\circ} \mathrm{E}\right), 2-10$.VI $2019,1{ }^{\top}$, AR; MSNR, quarter $408\left(54.750^{\circ} \mathrm{N}, 43.200^{\circ} \mathrm{E}\right)$, 2-10.VI 2019, 1 ô, AR.

DISTRIBUTION. Russia (first record). - Europe.

\section{*Lonchaea baechlii MacGowan, 2016}

MATERIAL. Russia: Republic of Mordovia, Temnikov distr., MSNR, quarter 299

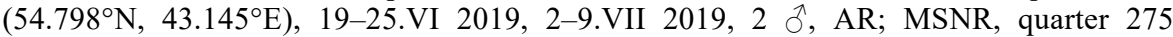
$\left(54.800^{\circ} \mathrm{N}, 43.160^{\circ} \mathrm{E}\right), 16-25 . \mathrm{VII} 2019,1{ }^{\lambda}$, AR; MSNR, quarter $427\left(54.736^{\circ} \mathrm{N}\right.$, $\left.43.220^{\circ} \mathrm{E}\right), 2-10$.VI 2019,1 今े, AR.

DISTRIBUTION. Russia (first record). - Europe (Serbia).

\section{**Lonchaea carpathica Kovalev, 1974}

MATERIAL. Russia: Republic of Mordovia, Temnikov distr., MSNR, quarter 302 $\left(54.791^{\circ} \mathrm{N}, 43.178^{\circ} \mathrm{E}\right), 2-10 . \mathrm{VI} 2019,47 \mathrm{\partial}^{\circ}, \mathrm{AR}$; MSNR, quarter $408\left(54.750^{\circ} \mathrm{N}, 43.200^{\circ} \mathrm{E}\right)$, 2-10.VI 2019, 10-19.VI 2019, 19-25.VI 2019, 25.VI-2.VII 2019, 2-9.VII 2019, 16-25. VII

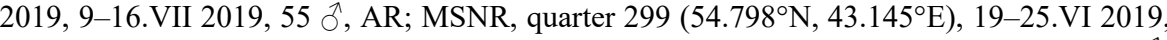
10-19.VI 2019, 25.VI-2.VII 2019, 2-9.VII 2019, 16-25.VII 2019, 9-16.VII 2019, 182 AR; MSNR, quarter $275\left(54.800^{\circ} \mathrm{N}, 43.160^{\circ} \mathrm{E}\right), 10-19 . \mathrm{VI} 2019,19-25 . \mathrm{VI} 2019,25$.VI-2.VII 2019, 2-9.VII 2019, 16-25.VII 2019, 49 $\hat{\jmath}$, AR.

DISTRIBUTION. Europe.

Lonchaea hackmani Kovalev, 1981

MATERIAL. Russia: Republic of Mordovia, Temnikov distr., MSNR, quarter 299 (54.798 $\left.{ }^{\circ} \mathrm{N}, 43.145^{\circ} \mathrm{E}\right), 2-9$.VII $2019,1 \mathrm{O}^{\lambda}, \mathrm{AR}$.

DISTRIBUTION. Northern and Central Europe.

\section{Lonchaea limatula Collin, 1953}

MATERIAL. Russia: Republic of Mordovia, Temnikov distr., MSNR, quarter 302 $\left(54.791^{\circ} \mathrm{N}, 43.178^{\circ} \mathrm{E}\right), 2-10 . \mathrm{VI} 2019,14{ }^{\circ}, \mathrm{AR}$; MSNR, quarter $299\left(54.798^{\circ} \mathrm{N}, 43.145^{\circ} \mathrm{E}\right)$, 19-25.VI 2019, 10-19.VI 2019, 2-9.VII 2019, 25.VI-2.VII 2019, 9-16.VII 2019, 16-25. 
VII 2019, $74 \mathrm{o}^{\lambda}$, AR; MSNR, quarter $275\left(54.800^{\circ} \mathrm{N}, 43.160^{\circ} \mathrm{E}\right), 10-19 . \mathrm{VI} 2019,19-25 . \mathrm{VI}$ 2019, 25.VI-2.VII 2019, 2-9.VII 2019, 16-25.VII 2019, 12 o, AR; MSNR, quarter 408 $\left(54.750^{\circ} \mathrm{N}, 43.200^{\circ} \mathrm{E}\right), 10-19 . \mathrm{VI} 2019,19-25 . \mathrm{VI} 2019,2-9$.VII $2019,9-16$. VII $2019,5{ }^{\uparrow}$, AR; MSNR, quarter $330\left(54.776^{\circ} \mathrm{N}, 43.183^{\circ} \mathrm{E}\right), 25 . \mathrm{VI}-2$.VII $2019,17 \mathrm{O}$, AR; MSNR, quarter $358\left(54.761^{\circ} \mathrm{N}, 43.196^{\circ} \mathrm{E}\right), 9-16 . \mathrm{VII} 2019,16-25 . \mathrm{VII} 2019,2$ ${ }^{\top}$, AR; MSNR, quarter 384 $\left(54.758^{\circ} \mathrm{N}, 43.215^{\circ} \mathrm{E}\right), 9-16 . \mathrm{VII} 2019,1$ 今े, AR.

DISTRIBUTION. Northern and Central Europe.

\section{Lonchaea sylvatica Beling, 1873}

MATERIAL. Russia: Republic of Mordovia, Temnikov distr., MSNR, quarter 299 $\left(54.798^{\circ} \mathrm{N}, 43.145^{\circ} \mathrm{E}\right), 25 . \mathrm{VI}-2$.VII 2019,1 § , AR.

DISTRIBUTION. Europe.

Lonchaea xylophila Kovalev, 1978

MATERIAL. Russia: Republic of Mordovia, Temnikov distr., MSNR, quarter 299 $\left(54.798^{\circ} \mathrm{N}, 43.145^{\circ} \mathrm{E}\right), 19-25 . \mathrm{VI} 2019,1$ ๙ , AR.

DISTRIBUTION. Russia (Moscow region). - Sweden.

\section{Family Psilidae}

\section{Psila fimetaria (Linnaeus, 1761)}

MATERIAL. Russia: Republic of Mordovia, Temnikov distr., MSNR, cordon Taratinsky $\left(54.743^{\circ} \mathrm{N}, 43.092^{\circ} \mathrm{E}\right), 26-29 . \mathrm{VI} 2020,1$ q, KT.

DISTRIBUTION. Russia (European part). - Europe, Algeria, Iran (Khaghaninia \& Gharajedaghi, 2014).

\section{**Chamaepsila bicolor (Meigen, 1826)}

MATERIAL. Russia: Republic of Mordovia, Temnikov distr., MSNR, cordon Novenkovsky $\left(54.931^{\circ} \mathrm{N}, 43.421^{\circ} \mathrm{E}\right), 4-7 . \mathrm{VII} 2020,1$ ${ }^{\lambda}, \mathrm{KT}$.

DISTRIBUTION. Records for Europe only were published (Andorra, Austria, Great Britain, Czech Republic, Denmark, Germany, Ireland, Slovakia, Switzerland, and Italy).

\section{Chamaepsila pallida (Fallén, 1820)}

MATERIAL. Russia: Republic of Mordovia, Temnikov distr., Purdoshki (54.669 $\mathrm{N}$, 43.542 $\left.{ }^{\circ} \mathrm{E}\right), 25$.VI 2020, 1 ${ }^{\lambda}$, KT.

DISTRIBUTION. Russia (European part). - Mongolia.

\section{Chamaepsila rufa (Meigen, 1826)}

MATERIAL. Russia: Republic of Mordovia, Temnikov distr., MSNR, cordon Novenkovsky $\left(54.931^{\circ} \mathrm{N}, 43.421^{\circ} \mathrm{E}\right), 4-7 . \mathrm{VII} 2020,1$ \%, KT.

DISTRIBUTION. Forest zone of the Palearctic (eastwards to Amur region in Russia).

\section{Loxocera fulviventris Meigen, 1826}

MATERIAL. Russia: Republic of Mordovia, Ichalki distr., National Park «Smolny», Barakhmanovskoe forestry, quarter $63\left(54.8331^{\circ} \mathrm{N}, 45.3796^{\circ} \mathrm{E}\right), 5 . \mathrm{IX} 2020,1$ \&, GS.

DISTRIBUTION. Forest zone of the Palearctic (eastwards to Amur region in Russia). 


\section{Family Strongylophthalmyiidae}

**Strongylophthalmyia pictipes Frey, 1935

MATERIAL. Russia: Republic of Mordovia, Temnikov distr., MSNR, cordon Novenkovsky $\left(54.931^{\circ} \mathrm{N}, 43.421^{\circ} \mathrm{E}\right), 4-7 . \mathrm{VII} 2020,1$ ๙, $\mathrm{KT}$.

DISTRIBUTION. Russia (European part, Siberia, Far East). - Europe.

\section{Family Ephydridae}

\section{Psilopa nigritella Stenhammar, 1844}

MATERIAL. Russia: Republic of Mordovia, Temnikov distr., MSNR, Pushta $\left(54.71^{\circ} \mathrm{N}\right.$, 43.22 $\left.{ }^{\circ} \mathrm{E}\right), 1-4 . \mathrm{IX} 2020,1$ ○, NV.

DISTRIBUTION. Russia (European part, Far East). - Europe, Northern Africa, Uzbekistan, Kazakhstan, China.

\section{Psilopa polita (Macquart, 1835)}

MATERIAL. Russia: Republic of Mordovia, Temnikov distr., MSNR, cordon Steklyanny $\left(54.894^{\circ} \mathrm{N}, 43.601^{\circ} \mathrm{E}\right), 9-15$. VII 2020,1 ô, KT.

DISTRIBUTION. Russia (European part, Siberia, Far East). - Europe, Northern Africa, Armenia, Georgia, Tajikistan, Kazakhstan, Korea, Japan.

NOTES. The record of this species from Mordovia (Plavilschikov, 1964) is confirmed by material examined.

\section{Hydrellia griseola (Fallen, 1813)}

MATERIAL. Russia: Republic of Mordovia, Temnikov distr., MSNR, Pushta $\left(54.71^{\circ} \mathrm{N}\right.$, 43.22 $\left.{ }^{\circ} \mathrm{E}\right), 1-5 . \mathrm{IX} 2020,1 \mathrm{O}, \mathrm{NV}$; MSNR, cordon Steklyanny $\left(54.894^{\circ} \mathrm{N}, 43.601^{\circ} \mathrm{E}\right), 9-15$. VII 2020, 1 क , KT; Purdoshki env. (54.689 $\left.\mathrm{N}, 43.533^{\circ} \mathrm{E}\right), 25 . \mathrm{VI} 2020,1{ }^{\lambda}$, NV .

DISTRIBUTION. Russia (European part, Siberia, Far East). - Cosmopolitan.

\section{Hydrellia obscura (Meigen, 1830)}

MATERIAL. Russia: Republic of Mordovia, Temnikov distr., MSNR, Pushta $\left(54.71^{\circ} \mathrm{N}\right.$,

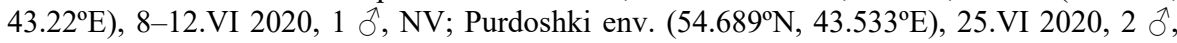
NV. 1996).

DISTRIBUTION. Russia (European part). - Europe, Northern Africa, Altai (Zatwarnicki,

\section{Dichaeta caudata (Fallen, 1813)}

MATERIAL. Russia: Republic of Mordovia, Temnikov distr., MSNR, Pushta $\left(54.72^{\circ} \mathrm{N}\right.$, 43.23ํㅡ), 8-21.V 2020, 1 §, NV.

DISTRIBUTION. Russia (European part, Siberia, Far East). - Holarctic.

Notiphila brunipes (Robineau-Desvoidy, 1830)

MATERIAL. Russia: Republic of Mordovia, Temnikov distr., Purdoshki env. (54.689 $\mathrm{N}$, 43.533E), 25.VI 2020, 1 , NV.

DISTRIBUTION. Russia (European part). - Europe. 


\section{Notiphila dorsata Stanhammar, 1844}

MATERIAL. Russia: Republic of Mordovia, Temnikov distr., Purdoshki (54.689 ${ }^{\circ}$,

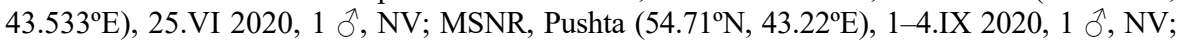
Saransk env. $\left(54.137^{\circ} \mathrm{N}, 44.907^{\circ} \mathrm{E}\right), 21 . \mathrm{VI} 2020,2$ Oे, NV.

DISTRIBUTION. Russia (European part, Caucasus, Ural, Far East). - Europe, Northern Africa, Armenia, China.

\section{Notiphila riparia Meigen, 1830}

MATERIAL. Russia: Republic of Mordovia, Temnikov distr., MSNR, Pushta $\left(54.71^{\circ} \mathrm{N}\right.$,

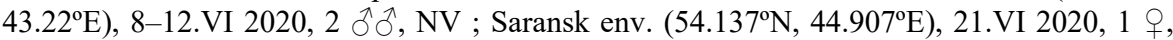
NV.

DISTRIBUTION. Russia (European part, Siberia, Far East). - Palaearctic.

\section{Ochthera mantis (DeGeer, 1776)}

MATERIAL. Russia: Republic of Mordovia, Temnikov distr., MSNR, Pushta $\left(54.71^{\circ} \mathrm{N}\right.$, $\left.43.22^{\circ} \mathrm{E}\right), 1-4 . \mathrm{IX} 2020,1$ \% , NV; Mordovia, Temnilov distr., Purdoshki $\left(54.669^{\circ} \mathrm{N}, 43.542^{\circ} \mathrm{E}\right)$, River Moksha, 25.VI 2020, 1 , KT.

DISTRIBUTION. Russia (European part, Siberia, Far East). - Holarctic.

\section{Discocerina obscurella (Fallen, 1813)}

MATERIAL. Russia: Republic of Mordovia, Temnikov distr., MSNR, Pushta $\left(54.71^{\circ} \mathrm{N}\right.$, 43.22 ${ }^{\circ} \mathrm{E}$ ), 1-4.IX 2020, 1 ㅇ, NV.

DISTRIBUTION. Russia (European part, Siberia, Far East). - Cosmopolitan

Hyadina rufipes (Meigen, 1830)

MATERIAL. Russia: Republic of Mordovia, Temnikov distr., MSNR, Pushta $\left(54.71^{\circ} \mathrm{N}\right.$, 43.22 ${ }^{\circ}$ E), 1-4.IX 2020, 1 q, NV.

DISTRIBUTION. Russia (European part, Far East). - Europe.

\section{Parydra aquila (Fallen, 1813)}

MATERIAL. Russia: Republic of Mordovia, Temnikov distr., Purdoshki $\left(54.74^{\circ} \mathrm{N}\right.$, $\left.43.67^{\circ} \mathrm{E}\right)$, Moksha bank, Salix, 27-29.VI 2020, 1 ․, KT; Purdoshki $\left(54.689^{\circ} \mathrm{N}, 43.533^{\circ} \mathrm{E}\right)$, 25.VI 2020, 1 $\hat{0}, \mathrm{NV}$

DISTRIBUTION. Russia (European part, Siberia, Far East). - Holarctic.

Coenia curvicauda (Meigen, 1830)

MATERIAL. Russia: Republic of Mordovia, Temnikov distr., MSNR, cordon Steklyanny $\left(54.8961^{\circ} \mathrm{N}, 43.6057^{\circ} \mathrm{E}\right), 8-13 . \mathrm{VII} 2020,1 \mathrm{\delta}^{\top}, \mathrm{KT}$.

DISTRIBUTION. Russia (European part, Siberia, Far East). - Holarctic.

\section{Coenia palustris (Fallen, 1823)}

MATERIAL. Russia: Republic of Mordovia, Temnikov distr., MSNR, Pushta $\left(54.72^{\circ} \mathrm{N}\right.$ $\left.43.23^{\circ} \mathrm{E}\right), 18-21 . \mathrm{V} 2020,1 \mathrm{O}^{\mathrm{N}}$, NV; MSNR, cordon Steklyanny $\left(54.8961^{\circ} \mathrm{N}, 43.6057^{\circ} \mathrm{E}\right), 8^{-}$ 13.VII 2020, 1 ㅇ, KT.

DISTRIBUTION. Russia (European part). - Europe, Azerbaijan, Kazakhstan, China. 
**Ephydra scholtzi Becker, 1896

MATERIAL. Russia: Republic of Mordovia, Temnikov distr., Purdoshki $\left(54.74^{\circ} \mathrm{N}\right.$, 43.67 E), Moksha bank, Salix, 27-29.VI 2020, 1 §, KT.

DISTRIBUTION. Russia (European part, Far East). - Europe.

\section{Paracoenia fumosa (Stenhammar, 1844)}

MATERIAL. Russia: Republic of Mordovia, Temnikov distr., MSNR, cordon Novenkovsky $\left(54.931^{\circ} \mathrm{N}, 43.421^{\circ} \mathrm{E}\right), 4-7$.VII 2020,3 ○, 1 으, KT; MSNR, Pushta $\left(54.72^{\circ} \mathrm{N}, 43.23^{\circ} \mathrm{E}\right)$,

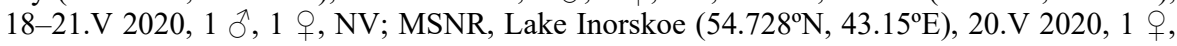
NV.

DISTRIBUTION. Russia (European part, Siberia, Far East). - Palaearctic.

\section{Setacera micans (Haliday, 1833)}

MATERIAL. Russia: Republic of Mordovia, Temnikov distr., MSNR, cordon Novenkovsky $\left(54.931^{\circ} \mathrm{N}, 43.421^{\circ} \mathrm{E}\right), 4-7$. VII $2020,1 \hat{\partial}, 1$ \% , KT.

DISTRIBUTION. Russia (European part, Eastern Siberia: Irkutsk region; Far East). Europe, Nearctic: Canada, USA (Alaska).

\section{Setacera trina Collin, 1963}

MATERIAL. Russia: Republic of Mordovia, Temnikov distr., MSNR, cordon Novenkovsky $\left(54.931^{\circ} \mathrm{N}, 43.421^{\circ} \mathrm{E}\right), 4-7 . \mathrm{VII} 2020,1$ ๙ , 1 q, KT.

DISTRIBUTION. Russia (European part). - Europe.

\section{Scatella obsoleta Loew, 1861}

MATERIAL. Russia: Republic of Mordovia, Temnikov distr., Purdoshki $\left(54.74^{\circ} \mathrm{N}\right.$,

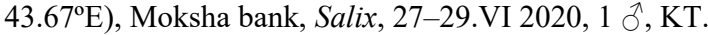

DISTRIBUTION. Russia (European part, Siberia, Far East). - Holarctic.

\section{Scatella paludum (Meigen, 1830)}

MATERIAL. Russia: Republic of Mordovia, Temnikov distr., Purdoshki $\left(54.6894^{\circ} \mathrm{N}\right.$,

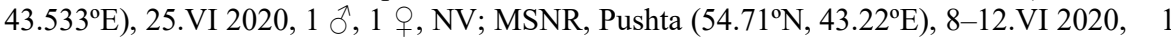

ô, 1 +, NV.

DISTRIBUTION. Russia (European part, Siberia, Far East). - Cosmopolitan.

\section{Scatella tenuicosta Collin, 1930}

MATERIAL. Russia: Republic of Mordovia, Temnikov distr., MSNR, Pushta $\left(54.71^{\circ} \mathrm{N}\right.$, 43.22 $2^{\circ}$ E), 8-12.VI 2020, 1 ㄱ, 1 ㅇ, NV.

DISTRIBUTION. Russia (European part, Siberia, Far East). - Cosmopolitan.

\section{Family Scathophagidae}

\section{Cleigastra apicalis (Meigen, 1826)}

MATERIAL. Russia: Republic of Mordovia, Temnikov distr., Purdoshki (54.6694 N, 43.5416 ${ }^{\circ}$ ), 25.VI 2020, 1 ㅇ, ME.

DISTRIBUTION. Russia (European part, Siberia, Far East). - Europe (widespread), Asia (Iraq, Mongolia). 


\section{Cleigastra leucostoma (Zetterstedt, 1846)}

MATERIAL. Russia: Republic of Mordovia, Temnikov distr., MSNR, River Pushta $\left(54.71^{\circ} \mathrm{N}, 43.22^{\circ} \mathrm{E}\right), 8^{8}-12 . \mathrm{VI} 2020,1$ ㅇ, NV; River Pushta $\left(54.75^{\circ} \mathrm{N}, 43.20^{\circ} \mathrm{E}\right), 25 . \mathrm{V} 2020,1$ +, $\mathrm{NV}$.

DISTRIBUTION. Russia (European part, Siberia, Far East). - Europe; North America.

\section{Cleigastra pilosa (Zetterstedt, 1838)}

MATERIAL. Russia: Republic of Mordovia, Temnikov distr., Purdoshki env. (54.698 ${ }^{\circ} \mathrm{N}$, $\left.43.533^{\circ} \mathrm{E}\right), 25$.VI 2020,1 ․ $\mathrm{NV}$.

DISTRIBUTION. Russia (European part, Siberia, Far East). - Northern and Central Europe, Mongolia.

\section{Cordilura albilabris (Fabricius, 1805)}

MATERIAL. Russia: Republic of Mordovia, Temnikov distr., MSNR, River Pushta $\left(54.71^{\circ} \mathrm{N}, 43.22^{\circ} \mathrm{E}\right), 8-12$.VI 2020,1 \% , NV.

DISTRIBUTION. Russia (European part, Siberia). - Europe, Azerbaijan.

\section{Cordilura albipes (Fallén, 1819)}

MATERIAL. Russia: Republic of Mordovia, Temnikov distr., MSNR, cordon Novenkovsky $\left(54.931^{\circ} \mathrm{N}, 43.421^{\circ} \mathrm{E}\right), 4-7 . \mathrm{VII} 2020, \mathrm{KT}$.

DISTRIBUTION. Russia (European part, Siberia, Far East). - Europe, Iran, Mongolia, Japan.

\section{Cordilura atrata (Zetterstedt, 1846)}

MATERIAL. Russia: Republic of Mordovia, Temnikov distr., MSNR, cordon Steklyanny $\left(54.894^{\circ} \mathrm{N}, 43.601^{\circ} \mathrm{E}\right), 12-15$.VII 2020,1 oे, KT.

DISTRIBUTION. Russia (European part, Siberia, Far East). - Europe, North America.

\section{Cordilura impudica (Rondani, 1867)}

MATERIAL. Russia: Republic of Mordovia, Temnikov distr., MSNR, Lake Inorskoe $\left(54.728^{\circ} \mathrm{N}, 43.15^{\circ} \mathrm{E}\right), 20 . \mathrm{V} 2020,1$ ते, NV.

DISTRIBUTION. Russia (European part, Siberia, Far East). - Europe, Kazakhstan.

\section{Cordilura picipes (Meigen, 1826)}

MATERIAL. Russia: Republic of Mordovia, Temnikov distr., MSNR, River Pushta $\left(54.75^{\circ} \mathrm{N}, 43.20^{\circ} \mathrm{E}\right), 21 . \mathrm{V} 2020,1{ }^{\AA}, \mathrm{NV}$.

DISTRIBUTION. Russia (European part, Siberia). - Europe, Mongolia.

\section{Cordilura rufipes (Meigen, 1826)}

MATERIAL. Russia: Republic of Mordovia, Temnikov distr., MSNR, River Pushta $\left(54.72^{\circ} \mathrm{N}, 43.23^{\circ} \mathrm{E}\right), 18-21 . \mathrm{V} 2020,2{ }^{\lambda}, \mathrm{NV}$; MSNR, River Pushta $\left(54.71^{\circ} \mathrm{N}, 43.22^{\circ} \mathrm{E}\right), 8_{-}$

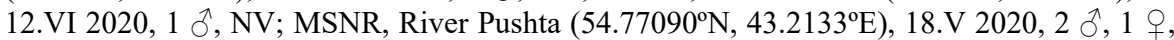
ME. China.

DISTRIBUTION. Russia (European part, Siberia, Far East). - Europe, Iran, Mongolia, 


\section{Norellisoma spinimanum (Fallén, 1819)}

MATERIAL. Russia: Republic of Mordovia, Temnikov distr., MSNR, River Pushta $\left(54.72^{\circ} \mathrm{N}, 43.23^{\circ} \mathrm{E}\right), 18-21 . \mathrm{V} 2020,1$ ㅇ, NV; MSNR $\left(54.7087^{\circ} \mathrm{N}, 43.2133^{\circ} \mathrm{E}\right), 10-13 . \mathrm{VII}$ 2020, 2 ऽ, 2 우, ME.

DISTRIBUTION. Russia (European part, Siberia, Far East). - Europe, Iran, Kazakhstan, Turkey, North America.

\section{Scathophaga furcata (Say, 1823)}

MATERIAL. Russia: Republic of Mordovia, Temnikov distr., MSNR, River Pushta $\left(54.72^{\circ} \mathrm{N}, 43.23^{\circ} \mathrm{E}\right), 18-21 . \mathrm{V} 2020,1$ oे, NV.

DISTRIBUTION. Russia (European part, Siberia, Far East). - Europe, Asia, North America.

\section{Scathophaga inquinata (Meigen, 1826)}

MATERIAL. Russia: Republic of Mordovia, Temnikov distr., MSNR, cordon Novenkovsky $\left(54.931^{\circ} \mathrm{N}, 43.421^{\circ} \mathrm{E}\right), 4-7 . \mathrm{VII} 2020,1 \stackrel{\mathrm{o}}{\mathrm{N}}, \mathrm{KT}$; MSNR $\left(54.7482^{\circ} \mathrm{N}, 43.2010^{\circ} \mathrm{E}\right), 21 . \mathrm{V}$ 2020, 1 ठै, ME.

DISTRIBUTION. Russia (European part, Siberia, Far East). - Europe.

\section{Scathophaga stercoraria Linnaeus, 1758}

MATERIAL. Russia: Republic of Mordovia, Temnikov distr., MSNR, Pushta $\left(54.71^{\circ} \mathrm{N}\right.$, 43.22 $\left.{ }^{\circ} \mathrm{E}\right), 18-22 . \mathrm{V} 2020,1$ 亏े, NV.

DISTRIBUTION. Russia (European part, Siberia, Far East). - Europe, Asia, North Africa, North America.

NOTES. The record of this species from Mordovia (Plavilschikov, 1964) is confirmed by material examined.

\section{Scathophaga spurca (Meigen, 1826)}

MATERIAL. Russia: Republic of Mordovia, Zubova Polyana distr., Zubova Polyana

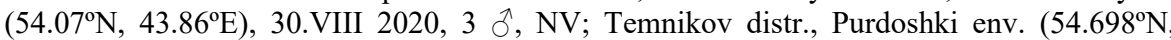

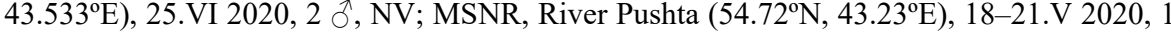
${ }^{\lambda}, \mathrm{NV}$; MSNR, cordon Taratinsky $\left(54.743^{\circ} \mathrm{N}, 43.092^{\circ} \mathrm{E}\right), 27-29$. VI 2020,2 。, 2 ㅇ, KT.

DISTRIBUTION. Russia (European part, Siberia, Far East). - Europe, Asia, North America.

\section{Scatomyza scybalaria Linnaeus, 1758}

MATERIAL. Russia: Republic of Mordovia, Temnikov distr., Purdoshki env. (54.689 ${ }^{\circ}$, 43.533ㄹ), 6.IX 2020, 1 ㅇ, NV

DISTRIBUTION. Russia (European part, Siberia, Far East). - Europe, Asia.

\section{Trichopalpus fraternus (Meigen, 1826)}

MATERIAL. Russia: Republic of Mordovia, Temnikov distr., Purdoshki env. (54.698 $\mathrm{N}$,

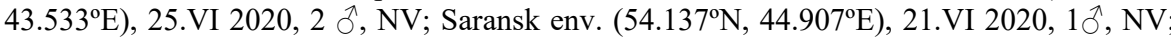
MSNR, cordon Novenkovsky $\left(54.931^{\circ} \mathrm{N}, 43.421^{\circ} \mathrm{E}\right), 4-7 . \mathrm{VII}$ 2020, 1 ㅇ, KT; MSNR, cordon Steklyanny $\left(54.894^{\circ} \mathrm{N}, 43.601^{\circ} \mathrm{E}\right), 9-15$.VII $2020,1 \mathrm{o}^{\top}, \mathrm{KT}$.

DISTRIBUTION. Russia (European part, Siberia, Far East). - Europe. 


\section{Trichopalpus punctipes (Meigen, 1826)}

MATERIAL. Russia: Republic of Mordovia, Temnikov distr., MSNR, River Pushta $\left(54.71^{\circ} \mathrm{N}, 43.22^{\circ} \mathrm{E}\right), 22-24 . \mathrm{VI} 2020,1$ ठ, $\mathrm{NV}$.

DISTRIBUTION. Russia (European part, Siberia, Far East). - Europe, Mongolia, North America.

\section{DISCUSSION}

A total of 55 Diptera species from eight families (Tanypezidae, Megamerinidae, Acroceridae, Psilidae, Lonchaeidae, Strongylophthalmyiidae, Ephydridae, and Scathophagidae) collected in the Republic of Mordovia, Russia are listed. The families Tanypezidae, Megamerinidae, Acroceridae, Strongylophthalmyiidae, and Psilidae are listed for the first time for the region's fauna. New to the fauna of Russia are Rotearomyia withersi MacGowan, 2014 and Lonchaea baechlii MacGowan, 2016 (Lonchaeidae). Megamerina dolium Fabricius, 1805, Lonchaea carpathica Kovalev, 1974, Ephydra scholtzi Becker, 1896, Strongylophthalmyia pictipes Frey, 1935, and Chamaepsila bicolor (Meigen, 1826) recorded for the center of the European part of Russia for the first time. Most of the discovered species are fairly common and widespread. In the Lonchaeidae Protearomyia withersi is known from across Europe where it is mainly associated with wood pasture habitats. Lonchaea baechlii was previously only known from the single male (holotype) from Serbia, the larvae are probably saprophagous. Lonchaea xylophila, whose larvae are associated with decaying birch (Betula sp.), is only known outside Russia from Sweden. In the Ephydridae, the recording of Setacera trina, previously only known in Russia from the Moscow region, is of interest, although the species has a fairly wide distribution in Europe (Bosnia and Herzegovina, Czech Republic, Germany, Great Britain, Italy, Poland, Slovakia) An unusual find is Ephydra scholtzi, whose larvae develop in salty water. This species is usually found in the coastal zone of the seas or in the waters of mineral springs.

\section{ACKNOWLEDGEMENTS}

The authors are grateful to A.I. Shatalkin and A.L. Ozerov (Zoological Museum of Moscow State University) for their help in identification of our material.

\section{REFERENCES}

Astakhov, D.M., Ruchin, A.B., Romadina, O.D. \& Pristrem, I.M. 2019. To robber flies fauna (Diptera: Asilidae) of Mordovia, Russia. Biodiversitas, 20(4): 994-1005. DOI: https://doi.org/10.13057/biodiv/d200409

Budaeva, I.A. \& Ruchin, A.B. 2014. To the fauna of blackflies (Diptera: Simuliidae) of the Republic of Mordovia (Russia). Caucasian Entomological Bulletin, 10(1): 155-159. [In Russian]

Buck, M. \& Marshall, S.A. 2006. Revision of New World Loxocera (Diptera: Psilidae), with phylogenetic redefinition of Holarctic subgenera and species groups. European Journal of Entomology, 103: 193-219.

Chursina, M.A. \& Ruchin, A.B. 2018a. A checklist of Bombyliidae (Diptera) from Mordovia, Russia and variation of wing shape in Bombylius species. Biodiversitas, 19(6): 21472156. DOI: https://doi.org/10.13057/biodiv/d190622

Chursina, M.A. \& Ruchin, A.B. 2018b. A checklist of Syrphidae (Diptera) from Mordovia, Russia. Halteres, 9: 57-73. DOI: https://doi.org/10.5281/zenodo.1255874 
Courtney, G.W., Pape, T., Skevington, J.H. \& Sinclair, B.J. 2009. Biodiversity of Diptera. P. 185-222. In: Foottit, R.G. \& Adler, P.H. (Eds.). Insect Biodiversity: Science and Society. Blackwell Publishing Ltd. DOI: https://doi.org/10.1002/9781444308211.ch9

De Jong, Y., Verbeek, M., Michelsen, V., Bjørn, P., Los, W., Steeman, F., Bailly, N., Basire, C., Chylarecki, P., Stloukal, E., Hagedorn, G., Wetzel, F., Glöckler, F., Kroupa, A., Korb, G., Hoffmann, A., Häuser, C., Kohlbecker, A., Müller, A., Güntsch, A., Stoev, P. \& Penev, L. 2014. Fauna Europaea - all European animal species on the web. Biodiversity Data Journal, 2: e4034. DOI: https://doi.org/10.3897/BDJ.2.e4034

Della Bella, V., Bazzanti, M. \& Chiarotti, F. 2005. Macroinvertebrate diversity and conservation status of Mediterranean ponds in Italy: water permanence and mesohabitat influence. Aquatic Conservation: Marine and Freshwater Ecosystems, 15: 583-600.

Dvořák, L., Dvořáková, K., Oboňa, J. \& Ruchin, A.B. 2020. Selected Diptera families caught with beer traps in the Republic of Mordovia (Russia). Nature Conservation Research, 5(4): 65-77. https://doi.org/10.24189/ncr.2020.057

Gornostaev, N.G. \& Sorokina, S.Yu. 2020. An annotated list of the drosophilid flies (Diptera: Drosophilidae) of Khakassia. Far Eastern Entomologist, 401: 13-17. DOI: https://doi.org/10.25221/fee.401.3

Grichanov, I.Ya. \& Khruleva, O.A. 2018. Fauna and ecology of Dolichopodidae (Diptera) from Wrangel Island Nature Reserve (Chukotka, Russia). Nature Conservation Research, 3(3): 37-45. DOI: http://dx.doi.org/10.24189/ncr.2018.023

Han, H.Y. \& Choi, D.S. 2001. Diptera (Syrphidae). Economic Insects of Korea 15. Insecta Koreana Supplement, 22: 1-224.

Khaghaninia, S. \& Gharajedaghi, Y. 2014. The first report of three genera and six species of rust flies (Diptera: Psilidae) from Iran. Biharean Biologist, 8(2): 102-105.

Krivosheina, N.P., \& Krivosheina, M.G. 2019. Saproxylic Diptera (Insecta) of the Lazovsky State Nature Reserve (Russia). Nature Conservation Research, 4(3): 78-92. DOI: http:// dx.doi.org/10.24189/ncr.2019.052 [In Russian]

Krivosheina, M.G. \& Ozerov, A.L. 2020. A review of the genus Setacera Cresson 1930 (Diptera, Ephydridae) of Russia. Zoologicheskij Zhurnal, 99(8): 900-910. [In Russian]

MacGowan, I. 2014. Three new species of Protearomyia McAlpine, 1962 (Diptera; Lonchaeidae) with a key to males of the Palearctic species. Zootaxa, 3796: 337-348. DOI: https://doi.org/10.11646/zootaxa.3796.2.6

MacGowan, I. 2020. Lonchaeidae online. Available at: http://lonchaeidae.myspecies.info (accessed 1 October 2020).

MacGowan, I. \& Bächli, G. 2016. New species of Lonchaeidae from Switzerland and Serbia with a revised checklist of the Swiss fauna. Mitteilungen der Schweizerischen Entomologischen Gesellschaft, 89: 169-176.

Mathis, W.N. \& Zatwarnicki, T. 1995. World Catalog of shore-flies (Diptera: Ephydridae). Memoirs on Entomology. International. 4. Gainesville, Associated Publishers. Florida. $424 \mathrm{p}$.

Nartshuk, E.P. 2013. Fauna Europaea: Acroceridae. In: Beuk, P. \& Pape, T.P. (2013) Fauna Europaea: Diptera: Brachycera. Fauna Europaea version 2017.06 Available at: https://faunaeu.org.

Ozerov, A.L. 2019. A review of the genus Trichopalpus Rondani, 1856 (Diptera: Scathophagidae) of Russia. Russian Entomological Journal, 28(4): 470-484. DOI: https:// doi.org/ 10.15298/rusentj.28.4.17

Ozerov, A.L. \& Krivosheina, M.G. 2015. A review of the genera Cleigastra Macquart, Gonarcticus Becker, Gonatherus Rondani, Hexamitocera Becker, Nanna Strobl, Orthacheta Becker and Spathephilus Becker (Diptera, Scathophagidae) of Russia. Zootaxa, 4012(2): 201-258. DOI: https://doi.org/10.11646/zootaxa.4012.2.1 
Ozerov, A.L. \& Krivosheina, M.G. 2020. A review of the genus Norellisoma Wahlgren, 1917 (Diptera: Scathophagidae) of Russia. Russian Entomological Journal, 29(1): 103-120. DOI: https://doi.org/10.15298/rusentj.29.1.15

Pilipenko, V.E., Ruchin, A.B. \& Semishin, G.B. 2020. Cranefly fauna (Diptera: Limoniidae, Pediciidae, Tipulidae) of the Republic of Mordovia, Russia. Biodiversitas, 21(1): 355369. DOI: https://doi.org/10.13057/biodiv/d210143

Plavilshchikov, N.N. 1964. A list of insect species found on the territory of the Mordovia State Nature Reserve. Proceedings of the Mordovia State Nature Reserve, 2: 105-134. [In Russian]

Roháček , J. 2016. Strongylophthalmyiidae, Tanypezidae and Megamerinidae (Diptera) in the Czech Republic and Slovakia: current state of knowledge. Acta Musei Silesiae Scientiae Naturalis, 65(1): 1-13. DOI: https://doi.org/10.1515/cszma-2016-0001

Ruchin, A.B., Egorov, L.V., Khapugin, A.A., Vikhrev, N.E. \& Esin, M.N. 2020. The use of simple crown traps for the insects collection. Nature Conservation Research, 5(1): 87108. DOI: https://dx.doi.org/10.24189/ncr.2020.008

Shatalkin, A.I. 2008. East Palaearctic and Oriental flies of the genus Psila Meigen (Psilidae). Archives of the Zoological Museum of Moscow State University, 49: 48-69. [In Russian]

Soós, Á. 1974. Taxonomische und faunistische Untersuchungen über die Psiliden (Diptera) aus der Mongolei. Annales historico-naturales Musei nationalis hungarici, 66: 241-250.

Tiunova, T.M. \& Makarchenko, E.A. 2020. Chironomid community (Diptera: Chironomidae) of temporary stream of Southern Primorye, Russian Far East. Far Eastern Entomologist, 414: 1-10. DOI: https://doi.org/10.25221/fee.414.1

Zatwarnicki, T. 1996. New records of Western Palaearctic shore flies (Diptera, Ephydridae). Annals of the Upper Silesian Museum. Entomology, 6-7: 107-125.

(C) Far Eastern entomologist (Far East. entomol.) Journal published since October 1994.

Editor-in-Chief: S.Yu. Storozhenko

Editorial Board: A.S. Lelej, S.A. Belokobylskij, M.G. Ponomarenko, V.A. Mutin, E.A. Beljaev, E.A. Makarchenko, A.V. Gorochov, T.M. Tiunova, M.Yu. Proshchalykin, S.A. Shabalin, V.M. Loktionov

Address: Federal Scientific Center of the East Asia Terrestrial Biodiversity (former Institute of Biology and Soil Science), Far East Branch of the Russian Academy of Sciences, 690022, Vladivostok-22, Russia.

E-mail: storozhenko@biosoil.ru web-site: http://www.biosoil.ru/fee 